\title{
On-line Life History Calendar and Sensitive Topics: A Pilot Study
}

\author{
Davide Morselli \\ Institute of Social Sciences \& Swiss National Centre of Competence in Research "LIVES" - \\ University of Lausanne, Switzerland \\ Annick Berchtold \\ Abiris: Software Engineering, Yens, Switzerland \\ Joan-Carles Suris Granell \\ Research Group on Adolescent Health, Institute of Social and Preventive Medicine (IUMSP), \\ Lausanne University Hospital, Lausanne, Switzerland \\ André Berchtold \\ Institute of Social Sciences \& Swiss National Centre of Competence in Research "LIVES" - \\ University of Lausanne, Switzerland
}

Correspondence regarding this article should be addressed to Davide Morselli at the Swiss National Centre of Competence in Research "LIVES", University of Lausanne, CH-1015, Lausanne, Switzerland. E-mail: davide.morselli@unil.ch 
Running Head: WEB LIFE HISTORY CALENDAR

Web-Based Life History Calendar and Sensitive Topics: A Pilot Study

\begin{abstract}
The use of the life history calendar (LHC) or the event history calendar as tools for collecting retrospective data has received increasing attention in many fields of social science and medicine. However, little research has examined the use of this method with web-based surveys. In this study, we adapted this method to an on-line setting to collect information about young adults' life histories, sexual behaviors, and substance use. We hypothesized that the LHC method would help respondents to date sensitive and non-sensitive events more precisely than when using a conventional questionnaire. We conducted an experimental design study comparing university students' responses to an on-line LHC and a conventional on-line question list. A test-retest design in which the respondents completed the survey again two weeks later was also applied to test the precision and reliability of the participants' dating of events. The results showed that whereas the numbers of sensitive and non-sensitive events were generally similar for the two on-line questionnaires, the responses obtained with the LHC were
\end{abstract}


more consistent across the two administrations. Analyses of the respondents' on-line behavior while completing the LHC confirmed that respondents used the LHC's graphic interface to correct and reedit previous answers, thus decreasing data errors.

Keywords: Life history calendar, event history calendar, retrospective data collection, on-line survey, sensitive data. 


\section{Web-Based Life History Calendar and Sensitive Topics: A Pilot Study}

On-line survey research has become increasingly common in recent years in many scientific disciplines. Although web-based surveys offer the advantages of reducing costs, providing data more quickly than other methods, being more flexible and allowing the easy implementation of filters or ad hoc questions, a number of unanswered questions have risen regarding the quality of the data collected on-line. Because on-line survey research is a relatively new and constantly changing field, its tools need to be tested to understand what data can be produced and whether its results are comparable to those of conventional interviewing modes. In the present study, we focus on the use of the web mode to collect retrospective and sensitive data by means of life history calendar (LHC) or event history calendar tools. These types of tools have received increasing interest in many disciplines within and beyond the social sciences. However, very little systematic research has investigated their application to an on-line setting. The present study addresses the lack of systematic methodological research regarding on-line LHCs by focusing on the application of this method to sensitive topics.

\section{The Use of Life History Calendar Methods}

With an LHC, the respondent is asked to report easily recallable life events to help with retrieving events that happened in other life domains and that might be more difficult to recall or date correctly. LHCs have been shown to be very useful for collecting retrospective data on respondents' life courses and complex sequences of events in different life domains (Freedman, Thornton, Camburn, Alwin, \& YoungDeMarco, 1988). The main advantage of retrospective methods is their ability to 
collect life course and longitudinal data with limited time and cost investments. However, these methods are more susceptible than prospective methods to measurement errors caused by memory bias. Methodological literature has shown that the use of LHCs and other visual supports for time mapping can improve the precision of autobiographical memories (Axinn, Pearce, \& Ghimire, 1999; Belli, 1998; Belli, Shay, \& Stafford, 2001; Van der Vaart, W, \& Glasner, 2007; Van der Vaart, 2004). These findings make LHCs appealing for a broad range of research, including health research (e.g., Danford \& Martyn, 2013; Miller, Steele, Bilgen, \& Belli, 2009; Williams, Neighbors, \& Jackson, 2003) and research on sexual behaviors (Fisher, 2013; Martyn \& Martin, 2003), abuse and violence (Yoshihama, Gillespie, Hammock, Belli, \& Tolman, 2005), and criminal activities (Sutton, 2010).

Standard LHC procedures rely on interactive interviews, during which the interviewer helps the respondent to remember events, make links between domains, and report correct dates; this procedure is beneficial for recalling events. However, methodological research on social desirability effects (i.e., the tendency to present oneself in a socially acceptable way) has noted that for sensitive topics, selfadministered methods are superior to interviewer-administered ones (Tourangeau \& Smith, 1996; Tourangeau \& Yan, 2007). For this reason, there may be advantages to making LHCs suitable for self-administration. In addition, the expanded use of internet surveys has increased the drive to design tools that can be administered online, especially when targeting young populations that may be more comfortable using web-based tools. In this pilot study, we investigate the performance of an on-line selfadministered LHC compared with a conventional on-line questionnaire.

Life History Calendars for Sensitive Questions 
According to Belli (1998), LHCs follow the same logic as autobiographical memory; consequently, they can help respondents recall and report past events. Events can be recalled in hierarchical order (e.g., from most important to least important), chronological order, or synchronic order (e.g., in relation to other events and episodes). LHC interviews follow these event-recall structures, allowing the respondent to make connections among events that occurred in different life domains.

Validation studies have shown the efficacy of LHCs compared with conventional questionnaires (CQs). For instance, Becker and Sosa (1992) showed that LHCs produce more consistent reports than CQs do, with less superimposition of mutually exclusive behaviors. Similarly, Engel, Keifer, \& Zahm (2001) suggested that LHCs generate more complete responses than traditional question lists do. Indeed, the visual nature of LHCs allows both the interviewee and the interviewer to spot incongruous answers in the data, thus reducing the number of unreported events. Test-retest reliability studies showed very high agreement for reported life events, such as marriages and migrations (Engel et al., 2001). Experimental comparisons between LHCs and CQs showed that adding a timeline to questionnaires enhanced data quality compared with the standard questionnaire procedure (Van der Vaart, 2004). Similarly, Belli, Shay, and Stafford (2001) compared an LHC with standardized state-of-the-art questionnaire-based interviewing methodologies and found that the LHC produced better data pertaining to the number of residential moves, periods of unemployment and cohabitation history.

It has also been argued that the LHC method is particularly suitable for collecting sensitive data and is less susceptible to social desirability bias (Luke, Clark, \& Zulu, 2011). For instance, Goldman et al. (1989) compared the use of LHCs and CQs for collecting information about contraceptive use and found that the LHC interviews 
yielded superior data on contraceptive use history. Yoshihama et al. (2005) obtained similar results; they found that compared with a CQ, an LHC elicited more reports of lifetime intimate partner violence. Sutton, Bellair, Kowalski, Hutcherson and Hutcherson (2011) showed that an LHC yielded reliable data on criminal activities. Research on adolescents has also used the LHC method to collect data on sensitive topics, such as sexual risk behaviors (Martyn \& Martin, 2003; Martyn, Reifsnider, \& Murray, 2006). According to Clark and Mathur (2012), LHCs are particularly suitable for sensitive questions because they place life events into broader contexts (e.g., life trajectories, relationship histories) and reduce the potential embarrassment about answering specific questions.

\section{Mode Effect on Sensitive Questions and Web Questionnaires}

Although the interviewer-respondent interaction is one of the main of the LHC method, it also represents one of its main limitations. LHCs are most commonly administered face-to-face or by telephone (e.g., Belli, Smith, Andreski, \& Agrawal, 2007), which may render the LHC method less appealing as survey research shifts towards web-based questionnaires and the use of mobile devices.

In addition, a consistent body of research on sensitive questions has shown that desirability bias is more likely when using administration modes that involve the presence of an interviewer, and the effect is particularly strong effect for face-to-face interviews (Kreuter, Presser, \& Tourangeau, 2008; Tourangeau \& Smith, 1996). It has been shown that self-administered questionnaires improve the accuracy of reports of the number of abortions (London \& Williams, 1990), drug and alcohol use (Gfroerer \& Kennet, 2014) and sexual risk behaviors (Schroder, Carey \& Vanable, 2003).

Although methodological research on the use of LHCs argues that calendar methods are less affected by desirability bias than CQs during face-to-face 
interviewing (Fisher, 2013), the administration mode may still have an important effect. Indeed, in a study on sexual behavior in adolescence in which the participants were asked to choose between self- and interviewer-administered LHCs, $85.7 \%$ of respondents opted to use the self-administered calendar (Martyn, 2009). Thus, there is still a good argument for offering a self-administration option when sensitive topics are concerned, even when using LHCs.

Among self-administered modes, web surveys have been shown to produce slightly more honest responses regarding minor illegal activities (Huang, 2006) and undesirable behaviors (Kreuter et al., 2008) than classic paper-and-pencil questionnaires do. Similar results were found for questions concerning sexual behaviors, birth control, and unprotected sex, although the difference between web and paper-and-pencil questionnaires was not dramatic (Uriell \& Dudley, 2009). This small difference between web and paper-and-pencil surveys does not appear to be related to age or to the respondents' abilities to use one mode or the other. Research conducted in schools also showed little difference between teenagers' responses to web versus paper-and-pencil questionnaires on sensitive topics such as sexual behaviors, abuse and drug use (Bates \& Cox, 2008; De Looij-Jansen, Petra, \& De Wilde, 2008; Vereecken \& Maes, 2006). It has been shown that digitalization per se is not sufficient to improve the amount of data collected on drug use (Livingston, Komro, \& Wagenaar, 2015) and other sensitive topics (Gnambs \& Kaspar, 2014). Web modes are superior to paper modes only in the sense that they provide increased anonymity and privacy. This being said, the internet may provide a greater perception of anonymity than other survey modes and thus may produce slightly more honest responses to sensitive questions (Christopherson, 2007; Lucia, Herrmann, \& Killias, 2007). 
Previous research comparing interviewer- and self-administered paper-and-pencil LHCs has shown that for data related to residential moves, the number of jobs, the number of children, the number of partners, and periods of unemployment, selfadministered LHCs can produce data of the same quality as interviewer-administered modes (Morselli et al., 2013). In other words, these results suggest that selfadministered LHCs can be used for non-sensitive topics. Regarding sensitive questions, we can expect that like CQs, self-administered LHCs give the respondent a greater sense of privacy and thus yield more genuine answers. We can also expect that the sense of privacy increases in web modes, a finding that has already been shown for CQs, and that internet-based administration of LHCs would yield more genuine answers to sensitive questions.

This being said, little research regarding the use of web-based LHCs has been conducted. Sage, Evandrou and Falkingham (2013) developed an on-line calendar to study migration trajectories. The tool is presented to the participant as a grid with time units in columns and events in rows. Instructions for completing the calendar are provided next to each type of event. Once the grid is clicked in correspondence with o indicate an event, a pop-up window asks for specific details (e.g., the start, end, and type of event). However, because each event is recorded on a single row, the grid can become very long, forcing the respondent to scroll up and down to see previous answers or left and right to locate the correct time unit. Although no methodological experiments regarding respondents' behaviors were conducted in relation to this tool, previous research on CQs has shown that scrolling may increase item non-response and that it requires extra effort on the part of the respondent (Couper, Traugott, \& Lamias, 2001). 
Kite and Soh (2004) proposed a different tool that requires the respondent to answer a series of direct questions presented by an automatic system that simulates the typical interviewer interaction used when LHCs are administered via telephone. This procedure should be able to reproduce the interviewer-respondent interaction and its ability to help the respondent recollect and report events while also offering the advantage of not including an actual physical interviewer. Although the idea is intriguing, its implementation has not been finalized to date, and no results on its feasibility are available.

One intermediate solution has been investigated by Glasner, van der Vaart, and Dijkstra (2014), who used an on-line device developed by the CenteRpanel and RAND. The visual structure of this LHC differs slightly from conventional paper-andpencil models. The calendar grid is relatively small, and a series of questions appears on the screen with interfaces for responses. Once the answer is provided, it is displayed on the grid in colored cells that correspond to the year and life domain. In contrast to Sage and colleagues' LHC, this tool requires a reduced amount of interaction with the calendar grid itself; instead, the calendar served primarily as a visual aid for the respondent. Glasner and colleagues' study is one of very few methodological designs aimed at directly testing the performance of an on-line LHC. The results of a probability-based internet panel of 1,451 respondents showed that the use of an LHC was associated with lower dropout rates and higher completion rates for family and unemployment events. In addition, compared with the CQ, the LHC produced better respondent evaluations.

Although these results are encouraging, no research has been conducted on the use of self-administered web-based LHCs for sensitive topics, such as sexuality and substance use, with young respondents. If previous research has indicated slightly 
better performance for web versus paper modes among young respondents, it is pivotal to understand whether, once the interviewer-respondent interaction is eliminated, webbased LHCs will be able to maintain their performance in terms of data completeness, particularly concerning sensitive information. It is also necessary to understand whether the benefits of LHCs over CQs in terms of the correct dating of events are consistent in web mode, as they are for telephone and self-administered pencil-andpaper interviews (e.g., Belli et al., 2007; Morselli et al., 2013).

In the present study, therefore, we were interested in exploring the differences between an on-line LHC and an on-line CQ for collecting information about sensitive topics concerning sexuality and substance use among young respondents. In particular, we hypothesized that:

H1. In line with the literature on paper-and-pencil and face-to-face uses of LHCs (Clark \& Mathur, 2012; Engel, Keifer, \& Zahm, 2001; Martyn, Reifsnider, \& Murray, 2006), the on-line LHC has a performance equivalent to or better than that of the online CQ in terms of the amount of sensitive data collected;

H2. By introducing visual support for time mapping, the LHC helps respondents to more accurately date events and thus yields more accurate responses than the CQ.

\section{Method}

Questionnaires

Two different questionnaires, an LHC and a CQ, were used in this study. Both the LHC and CQ had the same three-part structure. The first and last parts of the questionnaires did not differ, and both questionnaires contained only two types of questions: multiple choice questions and questions that asked for the year a specific event occurred (see Figure 1). 
In contrast, the second part of the questionnaire was presented as a graphical calendar in the LHC condition and as a traditional questionnaire in the CQ condition. The LHC tool was designed ad hoc for this study. It was automatically generated, and it adapted to the respondent's age and sex based on the answers provided in the first part of the questionnaire. Each year on the grid was divided into the four seasons to enhance the precision of the answers (Figure 2). A number of time-related events that were asked about in the first part of the questionnaire were also automatically projected onto the calendar grid. In particular, the calendar displayed the year of the participant's first diet for weight loss, if any; the year that the participant completed compulsory schooling; the participant's first year of university; the year the participant obtained his/her first driver's license; the year the participant acquired his/her first smart-phone; and the year of first sexual intercourse (Figure 2). Each event appeared as a question-specific icon.

Similar to the LHC developed by RAND (Glasner et al., 2014), once the LHC was generated, a series of questions appeared at the left side of the screen. Sample questions were "If you have changed homes in your life, when did you move?"; "If you have smoked cigarettes, when did you smoke for the first time?"; and "If you have had unwanted sexual intercourse, when did it happen the first time?". In contrast with RAND's model, our respondents answered directly on the calendar grid, as in the paper-and-pencil versions. Each answer was displayed using an icon on the calendar grid, which allowed the respondent to see time sequences and view each event in relation to other reported events. In addition, the respondents could also edit, move in time or delete their previous answers at any point by clicking on any of the events displayed on the grid (Figure 3). 
FIGURE 3 ABOUT HERE

Depending on the questions, the answer could be unique (example: the first episode of drunkenness) or multiple (the time of each HIV test). Once they had finished a question, the respondents could move to the next one, but they also had the freedom to go back to previous questions if they thought they had forgotten something or to complete the calendar grid independently from the question sequence on the left side. For clarity, the questions were divided into eight domains (school, family, home, transition, other events, health, substances, sexual experiences), and the grid contained a different row to display the events in each domain (Figure 4). This solution aimed for a relatively compact calendar format to minimize vertical scrolling. Horizontal scrolling was still necessary to browse among the years.

FIGURE 4 ABOUT HERE

This LHC questionnaire was developed in Java (1.8), using the Spring webmvc (v. 4.0.3.release), Dojo (v. 1.9.3) and Hibernate (v. 4.3.5) software architectures. Apache Tomcat (v. 7.0) was used as the application server, and the software was hosted on a server at the University of Lausanne.

The CQ and a feedback questionnaire were developed on LimeSurvey v. 1.91+ on a server hosted by the University of Lausanne. The CQ included the same questions as the LHC, but the respondents were asked to type the years of the events in an empty cell (one per event) at the end of each question. The feedback questionnaire included questions about the type of browser used to access the questionnaire, problems encountered (whether technical or more general), and preferences about the type of questionnaire (LHC or CQ).

Data Collection 
Undergraduate students at the Universities of Lausanne and Geneva were solicited to take part in the study. Unlabeled sealed envelopes were randomly distributed to students who were attending a presentation about the study during one of their courses. The envelopes contained an information sheet about the study, the internet link to the questionnaire, and a unique identifier. The students who received envelopes were entitled to a gift card for 15 Swiss francs (approximately 15 US\$) that was given unconditionally without checking whether the student actually participated in the study. In total, 329 envelopes were distributed.

The participants were asked to complete two questionnaires: the first one was administered between December 1 and December 7, 2014 (Time 1) and the second one between December 15 and December 19, 2014 (Time 2). The participants were randomly assigned to four groups: one group completed the LHC twice, one group completed the CQ twice, and the other two groups completed either the LHC or the CQ at Time 1 and the other version at Time 2. After completing the second questionnaire, the students were asked to complete a very short feedback questionnaire that aimed to collect their comments and feelings about the two types of questionnaires. The whole study was entirely anonymous, and it was made clear to the students that the researchers would have no way to link a particular answer to a particular individual.

\section{Measures}

Both sensitive and non-sensitive data were collected with both the LHC and the CQ. Among the non-sensitive topics, the questionnaire asked about both multiple events (residential moves, repetitions of school years, children's birth years, deaths of family members) and unique events (year of parents' separation, year of leaving the parents' household). The questionnaire also asked about sexuality, including multiple 
events (years of sexual education classes, years of HIV tests) and unique events (vaccination against human papillomavirus [HPV], age at menarche, first use of hormonal contraception, first use of the day-after pill, first incident of sexual abuse, first pregnancy or partner's first pregnancy). Questions on substance use concerned the time of the first incident of smoking, getting drunk and using cannabis.

\section{Analytical Strategy}

Six completeness indicators were calculated: the number of total reported events, the number of multiple events, the number of unique events, the number of residential moves, the number of sexual education classes, and the number of HIV tests. Repeated school years, children's births, and deaths of family members were insufficiently common among the targeted population to be analyzed separately and therefore were only used to determine the total number of events and the number of multiple event indicators. Similarly, certain unique events were too rare be statistically analyzed and thus were only used to calculate the total number of events and unique event indicators.

The average completeness indicator scores for the LHC and the CQ were compared, and statistical significance was assessed with independent sample t-tests. In addition to the completeness indicators, we also analyzed the differences between the two questionnaires on the reported average dates of first occurrence of sexual behaviors and substance use. If one of the two tools was more affected by desirability bias, we would expect the respondents to report later occurrences of these types of events because that would be more socially acceptable among older populations.

Test-retest reliability analysis was conducted to explore the two tools' ability to yield consistent answers between the first and second administrations. Cohen's (1960) kappa $(k)$ was used to assess the coherence of the years for each event reported at Time 
1 and Time 2. The calculation of $k$ differed slightly for multiple events than for unique events. For the former, the reliability was assessed for the respondent's full trajectory to test whether the respondent reported the same sequence of years for each variable. For single events, only the coherence of the response at the two time points was used. Using this second method, it was also possible to compute the weighted $k$ (Cohen, 1968), which in the case of non-matching answers at two time points takes into account the distance between the answers. The percentage of exactly matching answers was also computed for both the LHC and the CQ.

All computations were performed using the R statistical environment (R Core team, 2014). The Type I error value was set to $5 \%$.

\section{Results}

Of the students who received a sealed envelope, 150 students (response rate 45.6\%) participated in the study. After insufficiently completed questionnaires were eliminated, 138 students completed the first questionnaire (Time 1, 65 LHC and 73 CQ), and 59 students completed the second questionnaire (Time 2, 25 LHC and 34 CQ). Table 1 shows the characteristics of the students who responded at Time 1 according to condition (LHC versus CQ). The two groups of respondents did not differ in terms of gender, age, country of birth, or overall self-reported health status. The two groups also had similar rates of sexual experiences and similar rates of being in an intimate relationship at the time of the study. Because the differences were not statistically significant, we can assume that the two groups in this study were fairly similar and comparable.

Table 2 reports the average scores for the six completeness indicators. Contrary to the literature showing that respondents report more events when answering calendar questionnaires (e.g., Engel, Keifer, \& Zahm, 2001), the overall number of collected 
events was lower in the LHC condition. The respondents who completed the CQ reported a higher number of multiple events, particularly sexual education classes. However, the numbers of other multiple events, such as residential moves or taking the HIV test, and the total number of unique events (leaving the family home, being vaccinated against HPV, first cigarette) did not differ significantly between the two questionnaires.

A closer look at the unique events showed that not only were there no statistically significant differences between the number of reported events but that the respondents in the two conditions reported statistically similar ages for their first events (Table 3).

Test-Retest Reliability

Among the 138 students who responded to the first questionnaire, 50 also completed the second questionnaire two weeks later (see Table 4). Twenty-four of them completed the same type of questionnaire they had completed at Time 1 (LHCLHC, and CQ-CQ conditions), while the remaining 26 completed the other type of questionnaire at Time 2 (LHC-CQ and CQ-LHC). Despite the very small sample, some reliability analyses could be performed. The results are reported in Table 5; in addition to Cohen's $k$ and weighted $k$ coefficients, the last two columns of Table 5 report the proportions of exactly matching data in the two conditions.

Concerning the number of events and the coherence of their sequences, the respondents who completed the $\mathrm{LHC}$ twice or the CQ twice gave the most coherent answers. The LHC-LHC and CQ-CQ conditions produced fairly similar results and had a moderate to high inter-wave reliability. However, the $k$ coefficient for years of sexual education classes was lower for the CQ.

In relation to reporting and dating single events, the highest $k$ 's were obtained in the LHC-LHC condition. In particular, for the first HIV test and for the first time 
smoking, using marijuana and using the day-after pill, all of the answers at Time 1 and 2 matched. In contrast, the LHC-LHC produced less reliable answers than the CQ-CQ for the dates of HPV vaccination and the age at menarche for girls, with only 60 and $77.8 \%$ of exact answers, respectively. However, the inter-wave reliability was higher when the weighted $k$ coefficient was considered, suggesting that the size of the error (i.e., the difference between the answers at Time 1 and 2) was relatively small. Concerning the year of first drunkenness, all of the conditions had relatively low raw $k$ coefficients. However, in both conditions that used the LHC, the weighted $k$ for the year of first drunkenness was large, again indicating small errors between Time 1 and Time 2. The weighted $k$ when the CQ was administered at Time 1 was smaller than when the first questionnaire was the LHC.

Low reliability was found when respondents switched questionnaire types. In particular, switching from the CQ to the LHC produced very low raw and weighted $k$ coefficients. In contrast, the LHC-CQ condition had low $k$ coefficients but good weighted $k$ values, again indicating small inter-wave incoherence.

Regarding the percentage of exactly matching events (same event and same timing) at Time 1 and Time 2, the results were clearly better for the LHC condition than for the CQ. For 11 of the 14 indicators, the percentage was higher among the respondents who completed the LHC twice compared with those who completed the CQ twice; the main exception was related to HPV vaccination.

Response Behavior and Task Appreciation

The respondents who completed the questionnaire at Time 2 were also asked to complete a short feedback questionnaire. Overall, the respondents who completed both types of questionnaires declared a preference for the LHC $(n=12)$ over the CQ $(n=$ 8). Most of the students who preferred the LHC were in the LHC-CQ condition ( $n=$ 
8), whereas students in the CQ-LHC condition expressed evenly distributed preferences for the two questionnaires (CQ $n=4$; $\operatorname{LHC} n=4)$.

In the LHC condition, the respondents' behavior was recorded to monitor the number of edits (i.e., modifications and deletions) that were performed ${ }^{1}$. Roughly half of the respondents who completed the LHC modified their answers at least once. In total, 255 events were modified during LHC completion; 77 of these events concerned sex-related behaviors, and 41 were related to substance use. Most of the respondents (66.7\%) who edited some answers expressed positive attitudes towards the LHC.

\section{Discussion}

This study aimed to explore the difference between a web-based LHC and a webbased CQ regarding sensitive topics among young respondents. Little methodological research has been conducted to date on on-line applications of the LHC (e.g., Glasner et al., 2014; Kite \& Soh, 2004), and to our knowledge, no study has directly addressed the impact of the on-line LHC in relation to sensitive topics. Thus, we were interested in whether the introduction of a visual support would encourage young respondents to report more events and whether it would enhance the precision of answers to sensitive questions.

Our results partially supported our first hypothesis (H1) by showing that the LHC and CQ were mostly equivalent. However, the results also showed that the on-line LHC did not outperform the CQ in terms of completeness. Against our expectations, a slightly smaller amount of data was collected with the LHC than with the CQ. The LHC yielded a lower number of reported events in relation to multiple event items

\section{1}

It was possible to track the respondents' behaviors only on the LHC platform. LimeSurvey, which was used to develop the CQ, does not have such a feature. 
(e.g., residential moves), for which the respondent had to indicate an open number of events. One explanation of this result is that completing the on-line LHC may have been more cognitively demanding than completing the CQ. The LHC may have encouraged the respondents to report fewer and less important events.

An alternative explanation is purely methodological: the structure of our on-line CQ may have been sufficient to encourage the respondents to provide more answers. Indeed, after each question that required multiple answers, a predetermined number of empty cells (from 5 to 10) was displayed. This feature may have primed the students that the "right" number of answers should have been close to the number of displayed cells, thus inciting respondents to provide more answers even if some were false, minor, or misdated. For instance, a higher number of sexual education classes was reported on the CQ, but the coherence of the answers between Time 1 and Time 2 was lower than that obtained with the LHC. The LHC may have limited the number of false or minor events reported and produced higher quality data in terms of correct dating.

The test-retest reliability analysis showed that both the LHC and the CQ produced consistent data when the respondents completed the same questionnaire twice. However, in line with our expectations (H2), the responses to the LHC were slightly more precise. The answers provided at the two time points were more congruent in the LHC-LHC condition than in the other conditions, supporting the hypothesis that the LHC facilitated the process of dating life events. This hypothesis is also supported by the higher coherence we observed between answers given on both the LHC and the CQ when the respondents completed the LHC first. Switching between the two types of questionnaires produced a higher number of incoherent answers. 
According to the literature (e.g., Belli, 1998), different cognitive processes are used to complete the LHC and the CQ when dating events. In our study, the LHC may have facilitated retrieval mechanisms by helping the respondents to visually map events and retain a memory of this map for a longer period than the CQ did; thus, the retest at Time 2 could have been influenced by the answers at Time 1 . If the influence of Time 1 on Time 2 is generally undesired in test-retest studies, in our case it could be actually an interesting result that shows the different cognitive processes that are required to complete the two questionnaires. In addition, and in line with previous studies (e.g., Glasner et al., 2014), the respondents expressed a slightly greater appreciation for the LHC than for the CQ. The LHC was particularly appreciated by respondents who completed it before they completed the CQ and by the respondents who took advantage of being able to edit events. This result supports Belli's (1998) hypothesis that the LHC facilitates a flexible mnemonic process, which may differ from the recollection process triggered by the CQ. Our version of the CQ was more directive and imposed a structured way of recollecting and reporting events (e.g., by indicating or priming a maximum number of events to report for multiple events). For the respondents who had appreciated the flexibility of the LHC at Time 1, the CQ might have appeared too constraining. Our data did not allow us to explore this hypothesis in depth, and it will need to be addressed in future studies.

Our findings showed good comparability of the on-line LHC with traditional web questionnaires for both sensitive and non-sensitive questions aimed at young respondents. In line with previous studies (Glasner et al., 2014; Morselli et al., 2013), our results suggest that the LHC is suitable for self-administration and web-based designs. Although this study focused on a very specific population and its results should be generalized to other populations with caution, our results showed that 
integrating LHC methods into internet surveys may be worth the effort when balancing advantages and disadvantages. Our on-line LHC collected only a slightly lower number of completed questionnaires (97\%) than the CQ did (99\%), and the LHC collected a slightly lower number of events; however, the LHC collected a higher quality of data in terms of precision and correct dating, supporting previous findings that showed that LHCs performed better than conventional methods for collecting retrospective data (e.g., Axinn, Pearce, \& Ghimire, 1999; Belli, 1998; Belli, Shay, \& Stafford, 2001; Van der Vaart, 2004).

Study Limitations and Future Developments

In addition to the promising results, this study also has some limitations that need to be considered when interpreting the findings. This experiment was conducted with a relatively small convenience sample of undergraduate students. Further studies should address this limitation by replicating this study with a more heterogeneous population to determine whether this web-based method is suitable only for certain categories of respondents (e.g., youth) or is generalizable to others.

This being said, we can suppose that even if the results might be related to the characteristics of our sample, they should respond to improvements in the usability of the on-line LHC. This study did not address the effect of the specific user interface we developed; however, we observed that the respondents who most appreciated the task of completing the LHC in our study were those who most often took advantage of the tools that allowed them to revise their answers as they completed the questionnaire. Making modifications easier and more intuitive can reduce the cognitive burden of completing the LHC. For instance, using drag-and-drop tools and other graphic and interactive applets may simplify the respondent's experience and increase the tools' performance and the data quality. The graphic display of questions and answers is 
indeed a significant aspect of self-administered questionnaires in general and of web questionnaires in particular (e.g., Couper, Tourangeau, Conrad, \& Crawford, 2004; Tourangeau, Couper, \& Conrad, 2004). This aspect is even more central in tools such as LHCs, which use the visual layout to facilitate the memory retrieval process. To date, little research has been developed in this direction, and there are no clear recommendations regarding the graphic aspects of web-based LHC interfaces. The development of more easily usable tools will most likely reduce the drawbacks that were observed in this study and increase the applicability of LHC methods to internet surveys. 


\section{References}

Axinn, W. G., Pearce, L. D., \& Ghimire, D. (1999). Innovations in life history calendar applications. Social Science Research, 28(3), 243-264.

Bates, S. C., \& Cox, J. M. (2008). The impact of computer versus paper-pencil survey, and individual versus group administration, on self-reports of sensitive behaviors. Computers in Human Behavior, 24, 903-916.

Becker, S., \& Sosa, D. (1992). An experiment using a month-by-month calendar in a family planning survey in Costa Rica. Studies in Family Planning, 23, 386391.

Belli, R. F. (1998). The structure of autobiographical memory and the Event History Calendar: Potential improvements in the quality of retrospective reports in surveys. Memory, 6(4), 383-406.

Belli, R. F., Shay, W., \& Stafford, F. (2001). Event History Calendars and question-list surveys: A direct comparison of interviewing methods. Public Opinion Quarterly, 65, 45-74.

Belli, R. F., Smith, L. M., Andreski, P. M., \& Agrawal, S. (2007). Methodological comparisons between CATI event history calendar and standardized conventional questionnaire instruments. Public Opinion Quarterly, 71(4), 603622.

Clark, S., \& Mathur, R. (2012). Dating, sex, and schooling in urban Kenya. Studies in Family Planning, 43(3), 161-174.

Cohen, J. (1960). A coefficient of agreement for nominal scales. Educational and Psychological Measurement.

Cohen, J. (1968). Weighted kappa: Nominal scale agreement provision for scaled disagreement or partial credit. Psychological Bulletin, 70(4), 213.

Couper, M. P., Tourangeau, R., Conrad, F. G., \& Crawford, S. D. (2004). What they see is what we get: Response options for web surveys. Social Science Computer Review, 22(1), 111-127. 
Couper, M. P., Traugott, M. W., \& Lamias, M. J. (2001). Web survey design and administration. Public Opinion Quarterly, 65(2), 230-253.

Christopherson, K. M. (2007). The positive and negative implications of anonymity in Internet social interactions:“On the Internet, Nobody Knows You're a Dog”. Computers in Human Behavior, 23(6), 3038-3056.

Danford, C. A., \& Martyn, K. K. (2013). Exploring eating and activity behaviors with parent-child dyads using event history calendars. Journal of Family Nursing, 1074840713491831.

De Looij-Jansen, V., Petra, M., \& De Wilde, E. J. (2008). Comparison of web-based versus paper-and-pencil self-administered questionnaire: Effects on health indicators in Dutch adolescents. Health Services Research, 43(5p1), 17081721.

Engel, L. S., Keifer, M. C., \& Zahm, S. H. (2001). Comparison of a traditional questionnaire with an icon/calendar based questionnaire to assess occupational history. American Journal of Industrial Medicine, 40, 502-511.

Fisher, C. M. (2013). Queering data collection: Using the life history calendar method with sexual-minority youth. Journal of Social Service Research, 39(3), 306321.

Freedman, D., Thornton, A., Camburn, D., Alwin, D., \& Young-DeMarco, L. (1988). The life history calendar: A technique for collecting retrospective data. Sociological Methodology, 18(1), 37-68.

Gfroerer, J., \& Kennet, J. (2014). Collecting survey data on sensitive topics: Substance use. In T. P. Johnson (Ed.) Health Survey Methods (pp. 447-472). Hoboken: John Wiley \& Sons, Inc.

Glasner, T., van der Vaart, W., \& Dijkstra, W. (2014). Calendar Instruments in Retrospective Web Surveys. Field Methods, 1525822X14554882.

Goldman, N., Moreno, L., \& Westoff, C. F. (1989). Collection of survey data on contraception: an evaluation of an experiment in Peru. Studies in Family Planning, 20, 147-157. 
Gnambs, T., \& Kaspar, K. (2014). Disclosure of sensitive behaviors across selfadministered survey modes: a meta-analysis. Behavior Research Methods, 47, 1237-1259.

Huang, H.-M. (2006). Do print and Web surveys provide the same results? Computers in Human Behavior, 22(3), 334-350.

Kite, J., \& Soh, L.-K. (2004). An Online Survey Framework Using the Life Events Calendar. CSE Technical reports, 104, University of Nebraska.

Kreuter, F., Presser, S., \& Tourangeau, R. (2008). Social desirability bias in CATI, IVR, and Web surveys the effects of mode and question sensitivity. Public Opinion Quarterly, 72(5), 847-865.

Livingston, M. D., Komro, K. A., \& Wagenaar, A. C. (2015). The Effects of Survey Modality on Adolescents' Responses to Alcohol Use Items. Alcoholism: Clinical and Experimental Research, 39(4), 710-715.

London, K., \& Williams, L. (1990). A comparison of abortion underreporting in an inperson interview and self-administered questionnaire. Paper presented at the Annual Meeting of the Population Association of America, Toronto.

Lucia, S., Herrmann, L., \& Killias, M. (2007). How important are interview methods and questionnaire designs in research on self-reported juvenile delinquency? An experimental comparison of internet vs paper-and-pencil questionnaires and different definitions of the reference period. Journal of Experimental Criminology, 3(1), 39-64.

Luke, N., Clark, S., \& Zulu, E. M. (2011). The relationship history calendar: improving the scope and quality of data on youth sexual behavior. Demography, 48(3), 1151-1176.

Martyn, K. K., \& Martin, R. (2003). Adolescent sexual risk assessment. Journal of Midwifery \& Women's Health, 48(3), 213-219.

Martyn, K. K., Reifsnider, E., \& Murray, A. (2006). Improving adolescent sexual risk assessment with event history calendars: A feasibility study. Journal of Pediatric Health Care, 20(1), 19-26. 
Miller, D. R., Steele, C. R., Bilgen, I., \& Belli, R. F. (2009, May). Health status measurement via event history calendars vs. conventional questionnaires: "Long time, no status change.". Paper presented at the $64^{\text {th }}$ Annual Conference of the American Association for Public Opinion Research, The Westin Diplomat Convention Center Hollywood, Florida.

Morselli, D., Spini, D., Le Goff, J.-M., Gauthier, J.-A., Brändle, K., Mugnari, E., ... Bumbaru, A. (2013). Assessing the performance of the Swiss Panel LIVES Calendar: Evidence from a pilot study. LIVES Working Papers, (2013-28). doi:10.12682/lives.2296-1658.2013.28

R Core Team (2014). R: A language and environment for statistical computing. $\mathrm{R}$ Foundation for Statistical Computing, Vienna, Austria. URL http://www.Rproject.org/.

Richman, W. L., Kiesler, S., Weisband, S., \& Drasgow, F. (1999). A meta-analytic study of social desirability distortion in computer-administered questionnaires, traditional questionnaires, and interviews. Journal of Applied Psychology, 84(5), 754.

Sage, J., Evandrou, M., \& Falkingham, J. (2013). Onwards or Homewards? Complex Graduate Migration Pathways, Well-being, and the "Parental Safety Net." Population, Space and Place, 19(6), 738-755.

Schroder, K. E., Carey, M. P., \& Vanable, P. A. (2003). Methodological challenges in research on sexual risk behavior: II. Accuracy of self-reports. Annals of behavioral medicine, 26(2), 104-123.

Sutton, J. E. (2010). A review of the life-events calendar method for criminological research. Journal of Criminal Justice, 38(5), 1038-1044.

Sutton, J. E., Bellair, P. E., Kowalski, B. R., Hutcherson, D. T., \& Hutcherson, D. T. (2011). Reliability and validity of prisoner self-reports gathered using the life event calendar method. Journal of Quantitative Criminology, 27, 151-171. 
Tourangeau, R., Couper, M. P., \& Conrad, F. (2004). Spacing, position, and order interpretive heuristics for visual features of survey questions. Public Opinion Quarterly, 68(3), 368-393.

Tourangeau, R., \& Smith, T. W. (1996). Asking sensitive questions the impact of data collection mode, question format, and question context. Public Opinion Quarterly, 60(2), 275-304.

Tourangeau, R., \& Yan, T. (2007). Sensitive questions in surveys. Psychological Bulletin, 133(5), 859.

Turner, C. F., Ku, L., Rogers, S. M., Lindberg, L. D., Pleck, J. H., \& Sonenstein, F. L. (1998). Adolescent sexual behavior, drug use, and violence: increased reporting with computer survey technology. Science, 280, 867-873.

Uriell, Z. A., \& Dudley, C. M. (2009). Sensitive topics: Are there modal differences? Computers in Human Behavior, 25(1), 76-87.

Vaart, V. der, W, \& Glasner, T. J. (2007). Applying a timeline as a recall aid in a telephone survey: a record check study. Applied Cognitive Psychology, 21, $227-238$.

Vaart, W. V. der. (2004). The timeline as a device to enhance recall in standardized research interviews: A split ballot study. Journal of Official Statistics, 20(2), $301-317$.

Vereecken, C. A., \& Maes, L. (2006). Comparison of a computer-administered and paper-and-pencil-administered questionnaire on health and lifestyle behaviors. Journal of Adolescent Health, 38(4), 426-432.

Williams, D. R., Neighbors, H. W., \& Jackson, J. S. (2003). Racial/ethnic discrimination and health: findings from community studies. American Journal of Public Health, 93(2), 200-208.

Yoshihama, M., Gillespie, B., Hammock, A. C., Belli, R. F., \& Tolman, R. M. (2005). Does the life history calendar method facilitate the recall of intimate partner violence? Comparison of two methods of data collection. Social Work Research, 29(3), 151-163. 
Table 1: Comparison of LHC and CQ at Time 1. All comparisons between the LHC and the CQ are non-significant at the 5\% level

\begin{tabular}{lcc}
\hline & LHC & CQ \\
& $(\mathrm{n}=65)$ & $(\mathrm{n}=73)$ \\
\hline Gender (female) & $79.4 \%$ & $79.2 \%$ \\
Age (years) & $21.7(4.3)$ & $21.4(4.6)$ \\
Country of birth (Switzerland) & $85.7 \%$ & $79.2 \%$ \\
Ever had sex & $83.9 \%$ & $88.9 \%$ \\
In a relationship & $67.7 \%$ & 60.3 \\
Experienced sexual abuse & $5.6 \%$ & $5 \%$ \\
Experienced own/partner's pregnancy & $4.2 \%$ & $5.3 \%$ \\
Self-reported health status: & & \\
$\quad$ Excellent & $7.9 \%$ & $15.3 \%$ \\
$\quad$ Very good & $49.2 \%$ & $48.6 \%$ \\
$\quad$ Good & $40.0 \%$ & $31.0 \%$ \\
$\quad$ Poor & $3.1 \%$ & $4.2 \%$ \\
$\quad$ Very poor & $0.0 \%$ & $1.4 \%$ \\
\hline
\end{tabular}


Table 2: Average Number of Events

\begin{tabular}{lccc}
\hline \multicolumn{3}{c}{ Means } \\
Indicator & LHC & CQ & t-test \\
& $(\mathrm{n}=65)(\mathrm{n}=73)$ & \\
& & & \\
\hline N. total events & 7.80 & 9.56 & $-2.46, \mathrm{p}=.02$ \\
N. multiple events & 3.63 & 5.09 & $-3.03, \mathrm{p}<.01$ \\
N. unique events & 4.17 & 4.47 & $-0.79, \mathrm{p}=.43$ \\
N. residential moves & 1.93 & 2.19 & $-0.71, \mathrm{p}=.48$ \\
N. sexual education classes & 1.12 & 2.00 & $-4.52, \mathrm{p}<.01$ \\
N. HIV tests & & & \\
\hline
\end{tabular}


Table 3: Average Age at First Event

\begin{tabular}{lccc}
\hline \multicolumn{3}{c}{ Means } \\
Indicator & LHC & CQ & t-test \\
& \multicolumn{3}{c}{$(\mathrm{n}=73$} \\
& $(\mathrm{n}=65)$ & \\
& & & \\
\hline First HIV test & 20.33 & 18.95 & $1.51, \mathrm{p}=.14$ \\
HPV vaccination & 15.06 & 16.19 & $-1.68, \mathrm{p}=.10$ \\
First time smoked & 15.48 & 16.06 & $-0.88, \mathrm{p}=.39$ \\
First time drank & 16.08 & 16.1 & $-0.05, \mathrm{p}=.96$ \\
First cannabis use & 17.26 & 16.64 & $1.29, \mathrm{p}=.20$ \\
Age at menarche & 13.02 & 13.13 & $-0.36, \mathrm{p}=.72$ \\
First hormonal & & & \\
contraception use & 17.39 & 18 & $-1.14, \mathrm{p}=.26$ \\
\hline First day-after pill & & & \\
\hline & 18.94 & 18.52 & $0.65, \mathrm{p}=.52$ \\
\hline
\end{tabular}


Table 4: Distribution of Respondents Who Answered Completely on Both Testing Occasions

\begin{tabular}{ccccc}
\hline & & \multicolumn{3}{c}{ Time 2 } \\
& & $\begin{array}{c}\text { LHC } \\
(\mathrm{n}=25)\end{array}$ & $\begin{array}{c}\text { CQ } \\
(\mathrm{n}=34)\end{array}$ & \\
\cline { 2 - 5 } Time 1 & LHC $(\mathrm{n}=65)$ & 10 & 15 & 25 \\
& CQ $(\mathrm{n}=73)$ & 11 & 14 & 25 \\
\cline { 2 - 5 } & & 21 & 29 & 50 \\
\hline
\end{tabular}


WEB LIFE HISTORY CALENDAR

Table 5: Test-Retest Reliabilities

\begin{tabular}{|c|c|c|c|c|c|c|c|c|c|c|}
\hline \multirow[b]{2}{*}{ Indicator } & \multicolumn{4}{|c|}{ Cohen's $k$} & \multicolumn{4}{|c|}{ Weighted $k$} & \multicolumn{2}{|c|}{$\begin{array}{l}\text { Exact matching } \\
\text { answers }\end{array}$} \\
\hline & LHC-LHC & LHC-CQ & CQ-LHC & CQ-CQ & LHC-LHC & LHC-CQ & CQ-LHC & CQ-CQ & LHC & CQ \\
\hline N. total events & .74 & .50 & .58 & .79 & - & - & - & - & $73.74 \%$ & $69.72 \%$ \\
\hline N. multiple events & .79 & .39 & .59 & .78 & - & - & - & - & $80.99 \%$ & $69.99 \%$ \\
\hline N. unique events & .70 & .61 & .57 & .79 & - & - & - & - & $73.48 \%$ & $71.05 \%$ \\
\hline N. residential moves & .76 & .63 & .74 & .88 & - & - & - & - & $97.61 \%$ & $98.45 \%$ \\
\hline $\begin{array}{l}\text { N. sexual education } \\
\text { classes }\end{array}$ & .81 & .13 & .31 & .60 & - & - & - & - & $99.13 \%$ & $95.96 \%$ \\
\hline N. HIV tests & 1.00 & .25 & .5 & .79 & - & - & - & - & $100 \%$ & $99.22 \%$ \\
\hline First HIV test & 1.00 & .20 & .33 & .68 & 1.00 & .81 & .67 & .98 & $100 \%$ & $78.57 \%$ \\
\hline HPV vaccination & .43 & .70 & .72 & .81 & .84 & .90 & .80 & .95 & $60 \%$ & $92.86 \%$ \\
\hline First time smoked & 1.00 & .60 & .72 & .68 & 1.00 & .64 & .95 & .87 & $100 \%$ & $78.57 \%$ \\
\hline First time drank & .64 & .44 & .37 & .51 & .98 & .96 & .56 & .83 & $70 \%$ & $57.14 \%$ \\
\hline First cannabis use & 1.00 & 1.00 & .41 & .60 & 1.00 & 1.00 & .93 & .96 & $100 \%$ & $64.29 \%$ \\
\hline Age at menarche & .69 & .56 & .45 & .81 & .75 & .96 & .88 & .99 & $77.78 \%$ & $83.33 \%$ \\
\hline $\begin{array}{l}\text { First hormonal } \\
\text { contraception }\end{array}$ & .88 & .90 & .65 & .81 & .98 & 1.00 & .69 & .99 & $90 \%$ & $85.71 \%$ \\
\hline First day-after pill & 1.00 & .63 & 1.00 & .83 & 1.00 & .97 & 1.00 & .98 & $100 \%$ & $85.71 \%$ \\
\hline
\end{tabular}




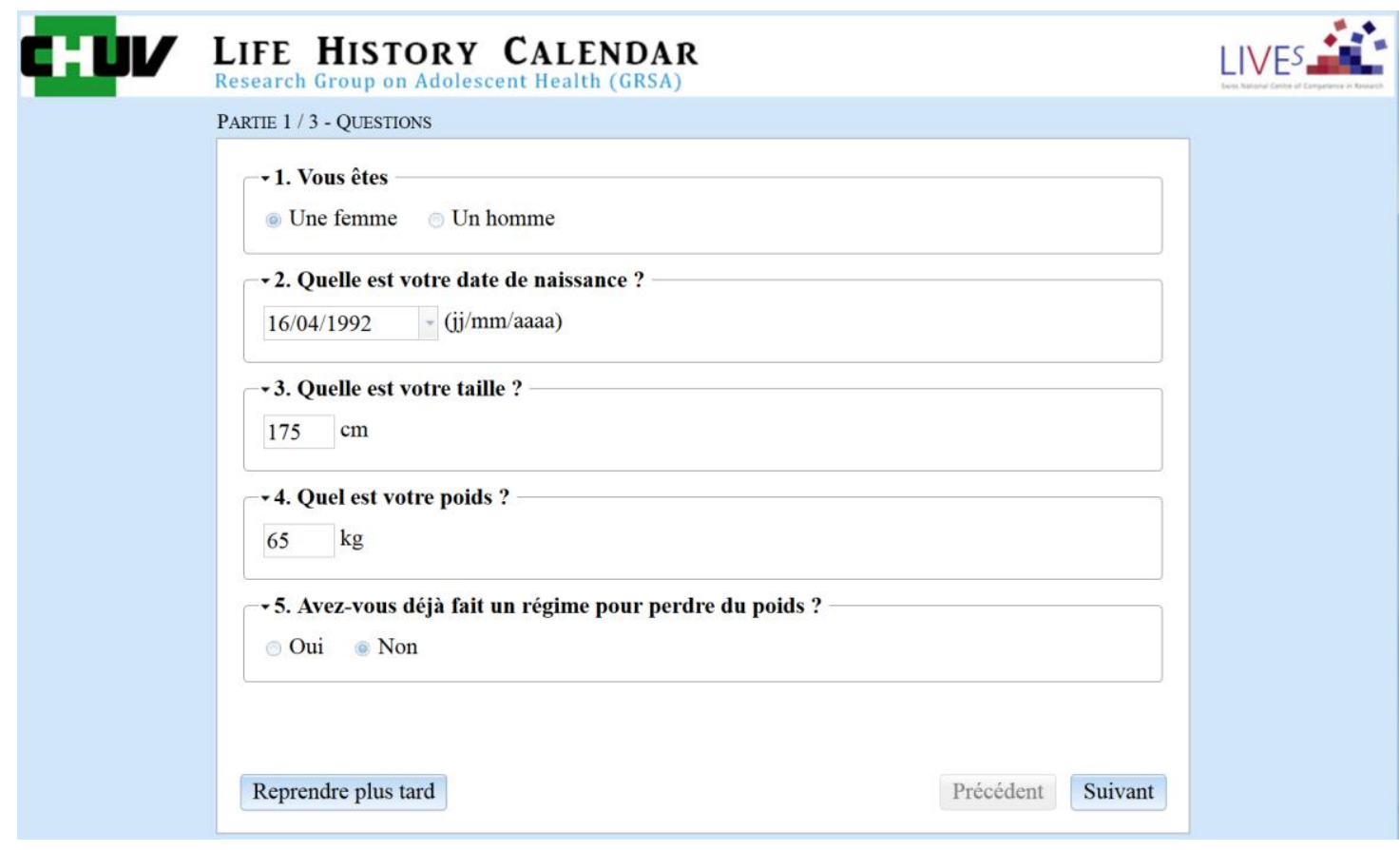

Figure 1: The first part of the LHC with socio-demographic questions such as gender and birth date. 


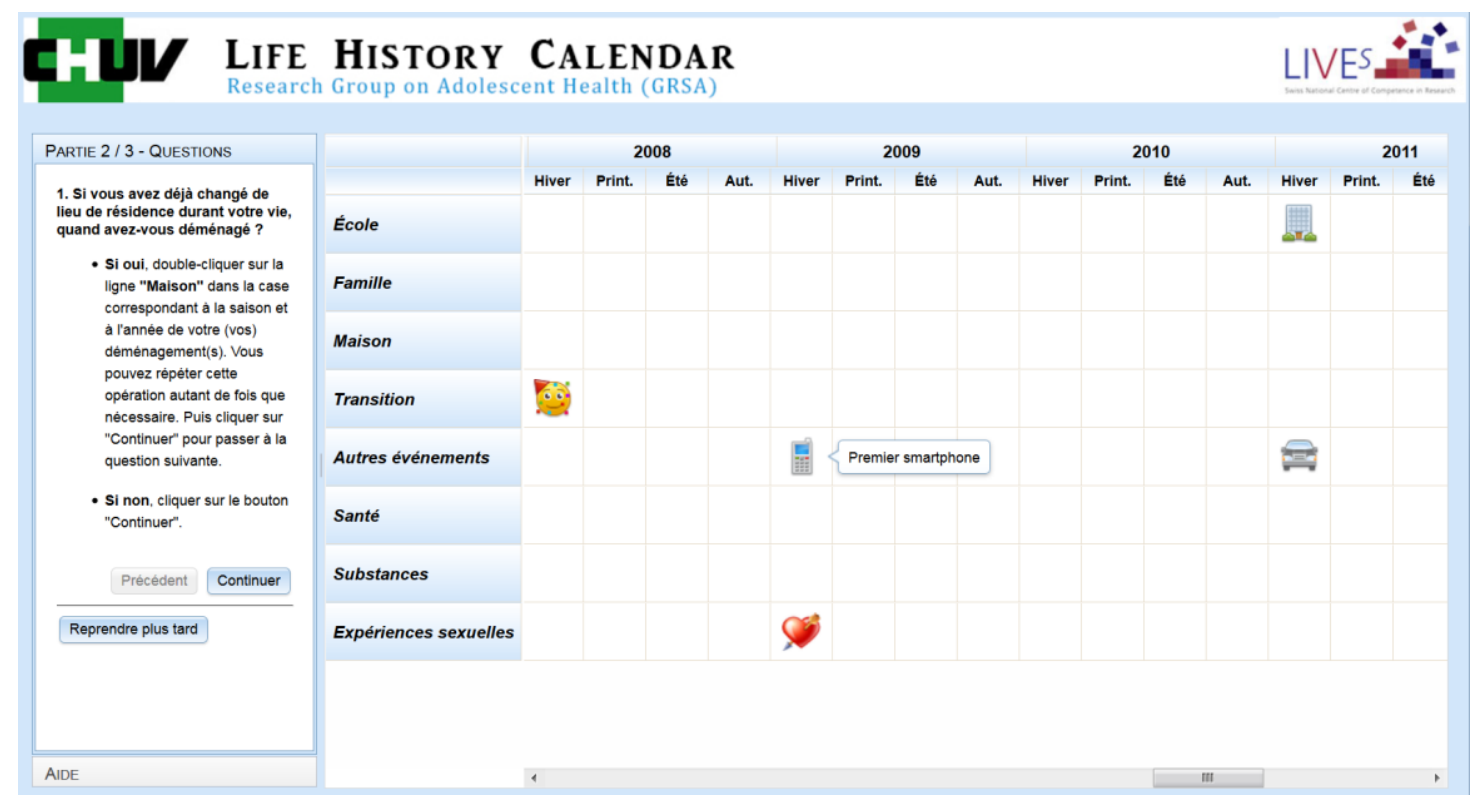

Figure 2: The second part of the LHC, with questions on the left and the grid with icons representing answers on the right. 


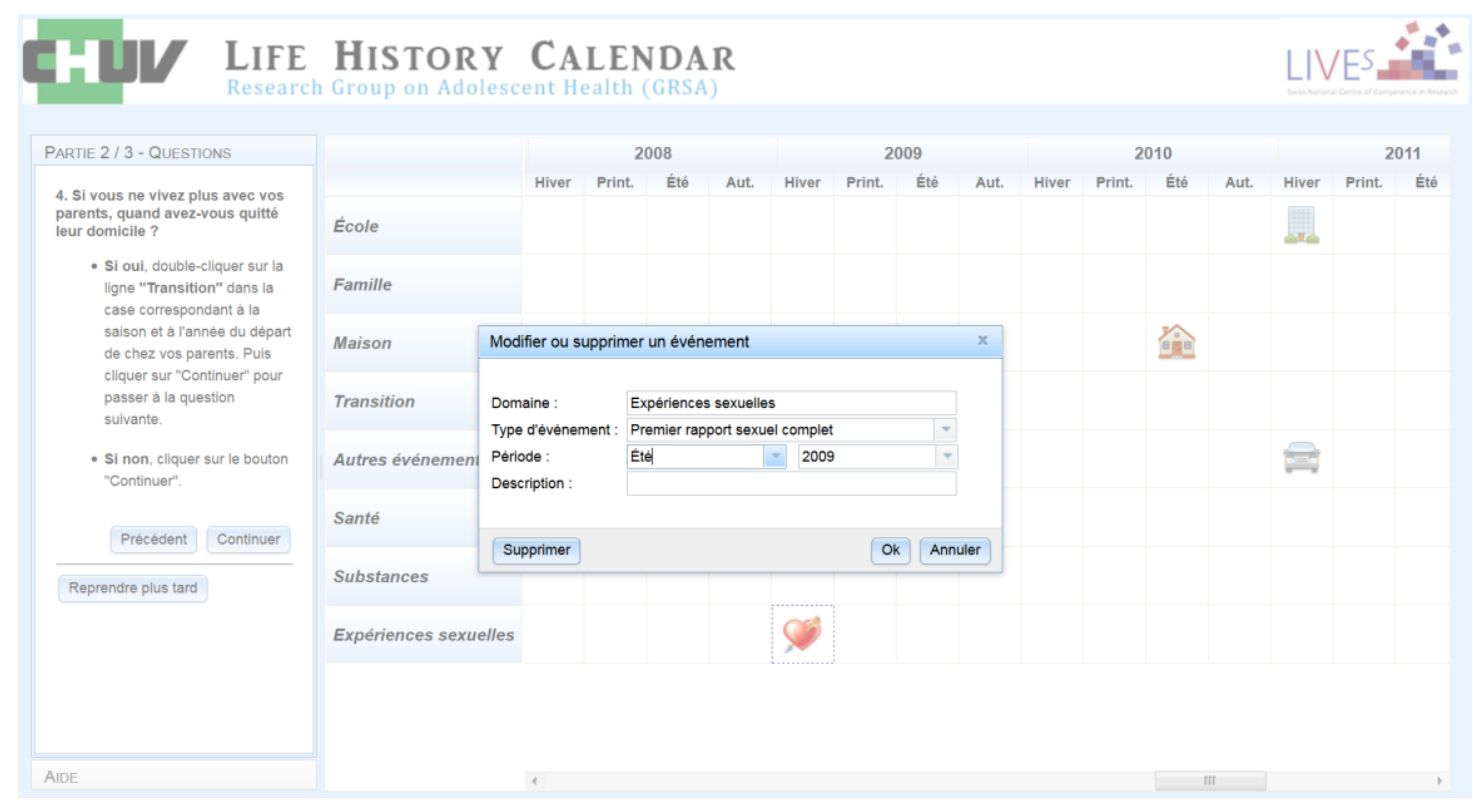

Figure 3: Editing of an event on the LHC grid. 


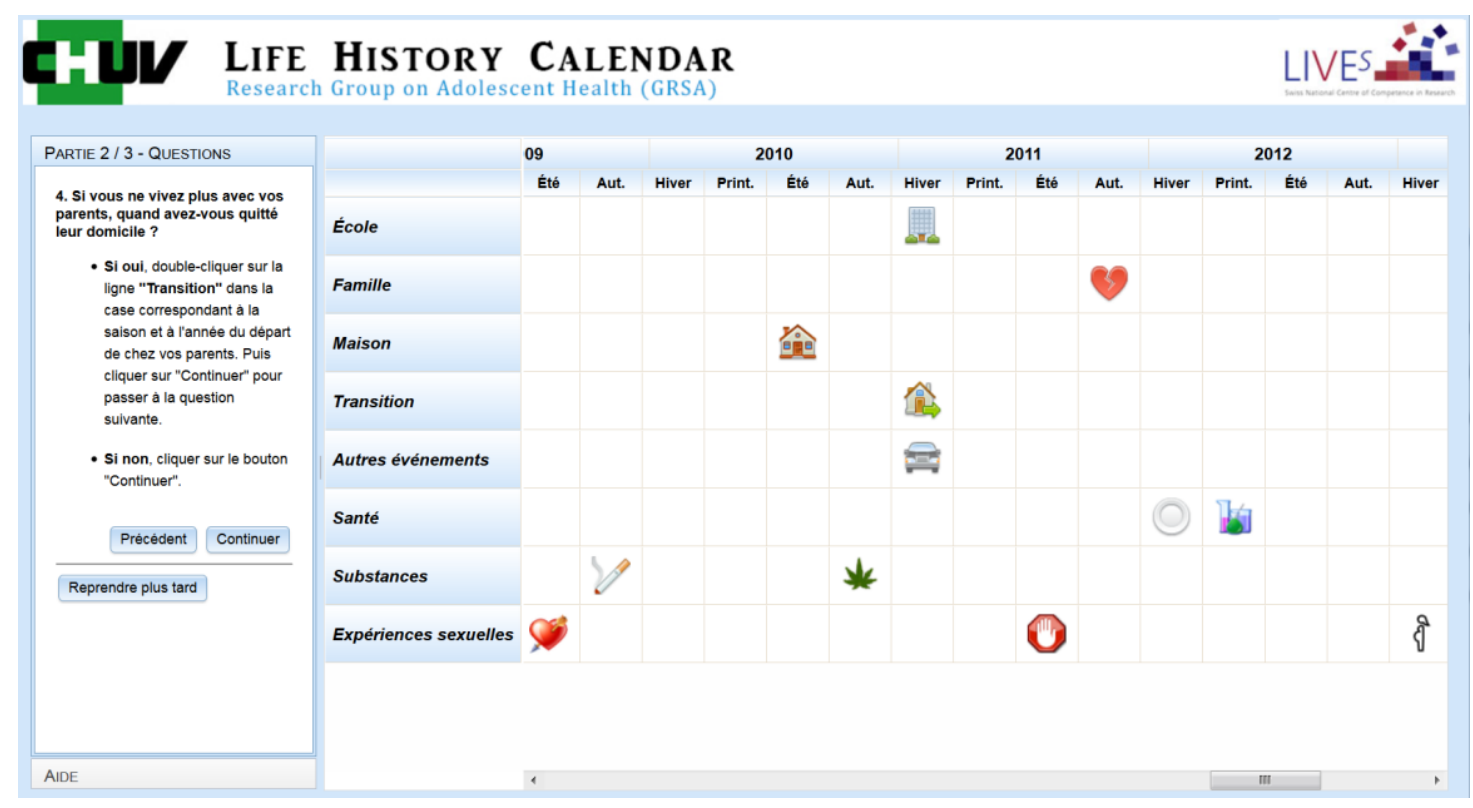

Figure 4: The second part of the LHC, with different events belonging to the eight domains. 